\title{
Disk evolution in the solar neighborhood
}

\section{Disk frequencies from 1 to $100 \mathrm{Myr}^{\star}$}

\author{
Álvaro Ribas ${ }^{1,2,3}$, Bruno Merín ${ }^{4}$, Hervé Bouy ${ }^{2}$, and Luke T. Maud ${ }^{5}$ \\ 1 European Space Astronomy Centre (ESA), PO Box 78, 28691 Villanueva de la Cañada, Madrid, Spain \\ e-mail: aribas@cab.inta-csic.es \\ 2 Centro de Astrobiología, INTA-CSIC, PO Box-Apdo. de correos 78, 28691 Villanueva de la Cañada Madrid, Spain \\ 3 Ingeniería y Servicios Aeroespaciales-ESAC, PO Box 78, 28691 Villanueva de la Cañada, Madrid, Spain \\ ${ }^{4}$ Herschel Science Centre, ESAC-ESA, PO Box 78, 28691 Villanueva de la Cañada, Madrid, Spain \\ 5 School of Physics \& Astronomy, EC Stoner Building, University of Leeds, Leeds LS2 9JT, UK
}

Received 3 September 2013 / Accepted 13 November 2013

\begin{abstract}
Aims. We study the evolution of circumstellar disks in 22 young (1 to $100 \mathrm{Myr}$ ) nearby (within $500 \mathrm{pc}$ ) associations over the entire mass spectrum using photometry covering from the optical to the mid-infrared.

Methods. We compiled a catalog of 2340 spectroscopically-confirmed members of these nearby associations. We analyzed their spectral energy distributions and searched for excess related to the presence of protoplanetary disks. The dataset has been analyzed in a homogeneous and consistent way, allowing for meaningful inter-comparison of results obtained for individual regions. Special attention was given to the sensitivity limits and spatial completeness of the observations.

Results. We derive disk fractions as probed by mid-infrared excess in the 22 regions. The unprecedented size of our sample allows us to confirm the timescale of disk decay reported in the literature and to find new trends. The fraction of excess sources increases systematically if measured at longer wavelengths. Disk percentages derived using different wavelength ranges should therefore be compared with caution. The dust probed at 22-24 $\mu \mathrm{m}$ evolves slower than that probed at shorter wavelengths (3.4-12 $\mu \mathrm{m})$. Assuming an exponential decay, we derive a timescale $\tau=4.2-5.8 \mathrm{Myr}$ at 22-24 $\mu \mathrm{m}$ for primordial disks, compared to $2 \sim 3 \mathrm{Myr}$ at shorter wavelengths $(3.4-12 \mu \mathrm{m})$. Primordial disks disappear around $10 \sim 20 \mathrm{Myr}$. Their decline matches in time a brief increase of the number of "evolved" disks (defined here as including transitional and debris disks). There is more dispersion in the fraction of excess sources with age when measured at $22-24 \mu \mathrm{m}$ in comparison to shorter wavelengths.

Conclusions. The increase in timescale of excess decay at longer wavelength is compatible with inside-out disk clearing scenarios. The increased timescale of decay and larger dispersion in the distribution of disk fractions at 22-24 $\mu \mathrm{m}$ suggest that the inner (terrestrial-planet forming) and outer (giant-planet forming) zones evolve differently, the latter potentially following a variety of evolutionary paths. The drop of primordial disks and the coincident rise of evolved disks at $10 \mathrm{Myr}$ are compatible with planet formation theories suggesting that the disappearance of the gas is immediately followed by the dynamical stirring of the disk.
\end{abstract}

Key words. protoplanetary disks - stars: formation - planetary systems - stars: pre-main sequence

\section{Introduction}

The structure and evolution of protoplanetary disks provide crucial information to better understand stellar and planetary formation (see Williams \& Cieza 2011, for a complete review on different aspects of protoplanetary disks evolution). The timescale of disk evolution, and its dependence on stellar age, mass, environment and multiplicity, is a fundamental parameter to constrain stellar formation theories and numerical simulations. The inter-phase between protoplanetary and debris disks is also key to understand the first stages of planetary formation. However, details of the processes involved in the dispersal of protoplanetary disks and the transition to debris disks have been elusive mostly because of the long distances to such disks (hence difficulty to resolved them) and the size of the samples studied so far.

Circumstellar disks are often detected via infrared (IR) excess over the stellar photosphere: dust in the disk is heated by

\footnotetext{
* Acknowledgements and Appendix A are available in electronic form at http://www . aanda. org
}

stellar radiation, and re-emits it in the mid/far-IR regime. The advent of IR observatories such as AKARI, the Spitzer Space Observatory, and more recently the Herschel Space Observatory produced very sensitive surveys over vast areas in several young nearby associations and clusters (Evans et al. 2009; Ishihara et al. 2010). Combined with all-sky or wide field surveys in the optical (e.g. CMC 14, and SDSS) and in the near/mid-IR (e.g. DENIS, UKIDSS, and WISE), they provide a sensitive and homogeneous photometric coverage of a large number of nearby regions and make a new and unique database for the study of protoplanetary disks.

We present a sample of 2340 young stars and brown dwarfs members of 22 nearby ( $<500$ pc) associations (13 star-forming regions and 9 young nearby associations) with ages ranging from 1 to 100 Myr. This sample of unprecedented size is a compilation of 37 spectroscopic studies published in the literature over the past two decades. The spectral energy distribution (SED) of each source was obtained by merging various catalogs covering the wavelength range from the optical ( $\sim V$-band) 
A\&A 561, A54 (2014)

Table 1. Young nearby regions (top) and associations (bottom) included in this study.

\begin{tabular}{|c|c|c|c|c|c|}
\hline Name & $\begin{array}{l}\text { Age } \\
(\mathrm{Myr})\end{array}$ & $\begin{array}{l}\text { Distance } \\
(\mathrm{pc})\end{array}$ & Membership and SpT & Spitzer photometry & Number of sources \\
\hline 25 Orionis & $7-10$ & 330 & (1) & (1) & 46 \\
\hline Cha I & 2 & $160-165$ & (2) (3) (4) & (3) (4) & 212 \\
\hline Cha II & $2 \pm 2$ & $178 \pm 18$ & (5) & (38) & 47 \\
\hline $\mathrm{CrA}$ & $1-3$ & $138 \pm 16$ & (6) (7) (8) (9) & (39) & 35 \\
\hline IC 348 & 2 & $285-315$ & (10) (11) & $(38)(40)$ & 298 \\
\hline$\lambda$ Orionis & 4 & $400 \pm 40$ & (12) & (41) & 114 \\
\hline Lupus & $1-1.5$ & $140-200$ & $(13)(14)(15)(16)$ & (38) & 217 \\
\hline NGC 1333 & 1 & $235 \pm 18$ & (17) & (42) & 74 \\
\hline Ophiuchus & $2-5$ & $120-145$ & (18) (19) (20) (21) & (38) & 258 \\
\hline$\sigma$ Orionis & $2-3$ & $440 \pm 30$ & (22) (23) (24) (25) (26) (27) (28) & (43) & 104 \\
\hline Serpens & 2 & $230 \pm 20$ & (17) (29) & (38) & 142 \\
\hline Taurus & $1-2$ & 140 & $(30)(31)(32)(33)$ & (32) (33) & 265 \\
\hline Upper Sco & $11 \pm 2$ & 140 & $(34)(35)(36)$ & (35) & 405 \\
\hline AB Dor & $50-100$ & 34 & (37) & $\ldots$ & 92 \\
\hline Argus & 40 & 106 & (37) & $\ldots$ & 51 \\
\hline$\beta$ Pic & 10 & 31 & (37) & $\ldots$ & 54 \\
\hline Carina & 30 & 85 & (37) & $\ldots$ & 37 \\
\hline Columba & 30 & 82 & (37) & $\ldots$ & 58 \\
\hline$\eta$ Cha & $4-9$ & 108 & (37) & $\ldots$ & 27 \\
\hline Octantis & 20 & 141 & (37) & $\ldots$ & 17 \\
\hline Tuc-Hor & 30 & 48 & (37) & $\ldots$ & 49 \\
\hline TW Hyа & 8 & 48 & (37) & $\ldots$ & 25 \\
\hline
\end{tabular}

Notes. Ages and distances are from Reipurth (2008a, b) unless specified. We also tabulate references to the different studies used to compile the sample of objects and to the Spitzer photometry, as well as the number of sources for each region.

References. (1) Hernández et al. (2007a); (2) Luhman (2007); (3) Luhman et al. (2008a); (4) Luhman \& Muench (2008); (5) Spezzi et al. (2008); (6) Neuhäuser et al. (2000); (7) Nisini et al. (2005); (8) Sicilia-Aguilar et al. (2008); (9) Sicilia-Aguilar et al. (2011); (10) Luhman et al. (2003); (11) Alves de Oliveira et al. (2013); (12) Bayo et al. (2011); (13) Krautter et al. (1997); (14) Allen et al. (2007); (15) Comerón (2008); (16) Mortier et al. (2011); (17) Winston et al. (2009); (18) Natta et al. (2002); (19) Wilking et al. (2005); (20) Alves de Oliveira et al. (2010); (21) Erickson et al. (2011); (22) Zapatero Osorio et al. (2002); (23) Muzerolle et al. (2003); (24) Barrado y Navascués et al. (2003); (25) Franciosini et al. (2006); (26) Caballero (2007); (27) Sacco et al. (2008); (28) Rigliaco et al. (2012); (29) Oliveira et al. (2009); (30) Luhman (2004); (31) Monin et al. (2010); (32) Rebull et al. (2010); (33) Rebull et al. (2011); (34) Preibisch et al. (2002); (35) Carpenter et al. (2006); (36) Lodieu et al. (2011); (37) Torres et al. (2008); (38) Evans et al. (2009); (39) Peterson et al. (2011); (40) Lada et al. (2006); (41) Barrado y Navascués et al. (2007); (42) Gutermuth et al. (2008); (43) Luhman et al. (2008b).

to the mid-IR $(22 \sim 24 \mu \mathrm{m})$. The quantity and the quality of the photometric measurements allow us to derive and study disk fractions in a consistent way for all the regions.

The present manuscript is the first of a series of articles where disk evolution will be investigated with a large and representative sample of young stars in the solar neighborhood. In Sect. 2, we present the sample and its characteristics. We describe the astrometric and photometric quality assessment and data curation process in Sect. 3. The SED fitting procedure is described in Sect. 4. In Sect. 5, we describe the method and criterion adopted to identify mid-IR excesses and the method used to derive excess (disk) fractions. The results are given in Sect. 6, and discussed in Sect. 7.

\section{Sample}

We compiled a sample of spectroscopically confirmed members of nearby regions using catalogs from the literature for the 25 Orionis, Chameleon I \& II, Corona Australis, IC 348, $\lambda$-Orionis, Lupus, NGC 1333, Ophiuchus, $\sigma$-Orionis, Serpens, Taurus, and Upper Scorpius associations and clusters. This original sample was then completed by adding the spectroscopically confirmed members of young nearby associations from the SACY survey presented in Torres et al. (2008), which includes the AB Doradus, Argus, $\beta$-Pictoris, Carina, Columba, $\eta$-Chamaeleon, Octantis, Tuc-Hor (THA), and TW Hya (TWA) associations. Table 1 summarizes of the basic properties of these regions, as well as the references used for the spectroscopically confirmed samples.

Young nearby associations offer several advantages for the study of stellar and planetary formation. Their distances and relative ages are in general relatively well known. Additionally, their proximity and youth make the detection of their least massive members easier, ensuring a complete (or at least representative) census of their stellar content from the most massive members to the substellar and planetary mass objects. As a result, they have extensively been studied over the past four decades (see e.g. Reipurth 2008a,b, and references therein). Additionally, nearby associations with ages in the range 5-80 Myr provide a good opportunity for studying the transition from protoplanetary to debris disks.

From the various catalogs found in the literature (see Table 1), we chose to keep only those objects with spectroscopically-obtained spectral type. This choice was motivated by two main reasons:

- spectroscopic confirmation minimizes contamination by unrelated foreground or background sources. Photometric surveys are known to include as much as $30 \%$ of contaminants (see e.g. Oliveira et al. 2009).

- knowing the spectral type breaks the degeneracy between $T_{\text {eff }}$ and visual extinction $\left(A_{V}\right)$ in the SED fitting, making the $A_{\mathrm{V}}$ estimation more robust.

A total of 2627 sources fulfill this first requirement. 


\subsection{Ages and distances}

Distances can be measured with different techniques, leading to several (sometimes inconsistent) values in the literature for the same region. With the exception of the SACY sample, most regions considered here are further away than $100 \mathrm{pc}$, and hence beyond Hipparcos' sensitivity limit (Perryman et al. 1997). Ages are difficult to determine, and star formation is probably not an abrupt event but extended in time (see e.g. Hartmann 2001). Absolute ages are usually uncertain and sometimes controversial (Bell et al. 2013), but the relative age sequence is expected to be accurate enough for the aim of our study and to perform a meaningful comparison of disk properties at different evolutionary stages (Soderblom et al. 2013).

For consistency, we chose to use ages and distances provided in Reipurth (2008a) and Reipurth (2008b) except when updated measurements were found in the literature:

- age of Cha II from Spezzi et al. (2008);

- distance to NGC 1333 from Hirota et al. (2008);

- age and distance to Upper Scorpius from Pecaut et al. (2012);

- age and distance to 25 Orionis come from Hernández et al. (2007a).

In the rest of our analysis, a conservative $50 \%$ uncertainty level was assumed for the ages, unless a different estimate was provided in the literature.

\subsection{Mass range covered by the sample}

The completeness level of the sample (in terms of mass or luminosity) is critical to assess the relevance of any statistical result, as an incomplete sample would yield biased results. This issue becomes even more important when mixing catalogs from different sources, which may have different completeness levels.

All the star-forming regions selected in this study have been extensively studied in the past (see i.e. Reipurth 2008a,b). The catalogs used to compile the sample presented here come from stellar population-oriented studies whose main objective was to define representative samples of members and derive accurate mass functions. On the high mass end, the various studies used in this analysis are expected to be complete since massive stars are bright and have been known for long. On the low mass end, the stellar population studies used in this analysis are in general complete or representative down the mid or late-M dwarfs. We therefore expect that our study is representative over the luminosity range between the most massive members (typically $\mathrm{O}, \mathrm{B}$ or A stars depending on the association) and the mid/late-M dwarfs.

\section{Astrometric and photometric quality assurance}

\subsection{Astrometry}

In the past, astrometric calibration was more complicated because of the lack of accurate reference astrometric catalogs. As a result, some of the oldest surveys used in this study have a poor astrometric accuracy. We undertook a data healing procedure to ensure consistent astrometric accuracy for all objects across the different regions and associations.

For the entire sample, we chose to use the 2MASS astrometry as reference (Skrutskie et al. 2006). 2MASS offers the advantage of homogeneously coverage of the entire sky, with an average astrometric accuracy of $\sim 0$. $^{\prime} 1$. In this process, each target is associated to the closest 2MASS counterpart within $3^{\prime \prime}$.
We a posteriori verify that the mean separation between the original and the 2MASS coordinates is smaller than 0.'4, except in the case of Ophiuchus (from Erickson et al. 2011) and Upper Sco (from Preibisch et al. 2002) where systematic offsets larger than $1^{\prime \prime}$ with respect to the 2MASS catalog were found and corrected.

Only 89 of the 2627 objects had no counterpart in 2MASS within $3^{\prime \prime}$ due to luminosities beyond the sensitivity limit of the survey. They were removed from the sample and ignored for the rest of the study.

\subsection{Photometry}

To include as much photometric information as possible for each source, we searched the literature and different public surveys for photometric measurements from the optical to the mid-IR.

\subsubsection{Surveys}

We retrieved information from WISE (Wright et al. 2010), Tycho-2 (Høg et al. 2000), the Sloan Digital Sky Survey Data Release 8 (SDSS DR8), UKIRT Infrared Deep Sky Survey (UKIDSS), DENIS (DENIS Consortium 2005), AKARI (Murakami et al. 2007), and the Carlsberg Meridian Catalog 14 (CMC 14). To take the different point spread function (PSF) sizes of each survey into account, we established an optimized search radius for each of them by fitting a Gaussian to the kernel density distribution of separations between the original (2MASS) and catalog coordinates. The search radius was then set to the average of the Gaussian $+3 \sigma$. As a sanity check, we verified that the search radius was consistent with the internal accuracy of the different surveys. When the number of sources was too small to derive a meaningful density distribution of separations, the search radius was set to $1^{\prime \prime}$. This value is a conservative compromise between the astrometric accuracy of the different surveys and the observed separation distribution. During this procedure, upper limits were treated with caution to include them as such. We chose to conservatively redefine as upper limits all fluxes with a signal-to-noise ratio $S / N<5$ in any 2MASS or WISE bands. We also quadratically added WISE absolute calibration errors of $2.4,2.8,4.5$ and $5.7 \%$ (respectively to its bands) as recommended in the WISE All Sky Catalog Explanatory Supplement.

\subsubsection{Optical to mid-IR photometry from the literature}

We complemented the all-sky optical surveys mentioned above with our own optical ( $r$ and/or $i$ ) photometry from the CanadaFrance-Hawaii Telescope, when available (Bouy et al., in prep.).

A number of associations presented in Table 1 have been observed with the Spitzer Space Observatory in the course of various legacy and other programs, and we included the corresponding IRAC and MIPS photometry. The Spitzer photometry proved to be extremely important for our study, since protoplanetary disks emit most of their light at mid-IR wavelengths. According to the Spitzer User Manual, absolute calibration of IRAC and MIPS fluxes is accurate to $\sim 5 \%$, and we add the corresponding error quadratically when not already included.

In both cases (optical and mid-IR photometry), a cross-match radius of $1^{\prime \prime}$ was used. 


\subsection{Resolved binaries and ultra-cool objects}

Multiple systems are frequent among stars (Lada 2006), and most of them are not resolved with the ground based or space (Spitzer and WISE) observations used in this work. The spectral type and photometry of such unresolved sources result in biased measurements. To minimize this problem, we performed an internal match for every region with a search radius of $2^{\prime \prime}$. A total of 68 objects (34 pairs, 2\% of the whole sample) were found and removed from the sample. Closer systems cannot be identified in the current dataset. We nevertheless note that the properties of multiple systems, and in particular their frequency and distribution of separation, are expected to remain largely unchanged after 1 Myr. Multiplicity frequency is also found to vary little between associations and clusters (Duchêne \& Kraus 2013) and should equally affect the various groups studied in this work. The statistics (and in particular the disk fractions) derived and discussed in the present study should therefore be regarded as statistics for the systems, rather than for individual sources.

Finally, we also removed objects with spectral types $\leq \mathrm{L} 0$. These objects, although very interesting for brown dwarf formation studies, are in most cases beyond the completeness limit of the various surveys used in our analysis. After this process, the sample is comprised of 2452 sources.

\section{Data processing}

\subsection{SED cleaning and $A_{V}$ fitting}

First, the spectral types were converted to $T_{\text {eff }}$ using:

- Schmidt-Kaler (1982) scale for spectral types K8 and earlier; - Luhman et al. (2003) scale for spectral types later than M0;

In spite of all the precautions described in Sect. 2, a blind crossmatch of large datasets inevitably contains errors and problems. We therefore visually inspected all the SEDs and rejected problematic sources and photometric measurements.

Extinction toward each source was then estimated by comparing the observed SEDs with a grid of reddened photospheric BT-Settl models from Allard et al. (2012) of the corresponding $T_{\text {eff }}$, and the extinction law of Indebetouw et al. (2005). Normalization of the observed and synthetic spectra was done at the 2MASS $J$ or $H$ band (in this order or priority) when present, and at the closest band otherwise. The photometric bands used for the $A_{\mathrm{V}}$ fitting were:

- all the optical bands, except those displaying obvious excesses. Some optical bands can indeed be strongly affected by emission lines related to accretion and outflows (Shu et al. 1994; Hartmann et al. 1994; Gullbring et al. 2000). This is specially the case for the $u$ band, which was systematically ignored in all fits;

- all the near-IR bands up to $K_{\mathrm{S}}$, included. Excess in these bands is rarer and usually moderate. We therefore chose to use all of them to maximize the number of measurements used for the fit. Small excesses at these bands result in slightly higher $A_{\mathrm{V}}$ values, but are in general tempered by the optical measurements and result mostly in a larger uncertainty.

Following Bayes theory, the best $\mathrm{A}_{\mathrm{V}}$ is obtained from the distribution of likelihood among all values computed over the grid. The explored $A_{\mathrm{V}}$ range was 0 to $20 \mathrm{mag}$ in steps of $0.1 \mathrm{mag}$. At least two photometric measurements in the mentioned wavelength range were required for the $A_{\mathrm{V}}$ to be fitted. For 10 sources,

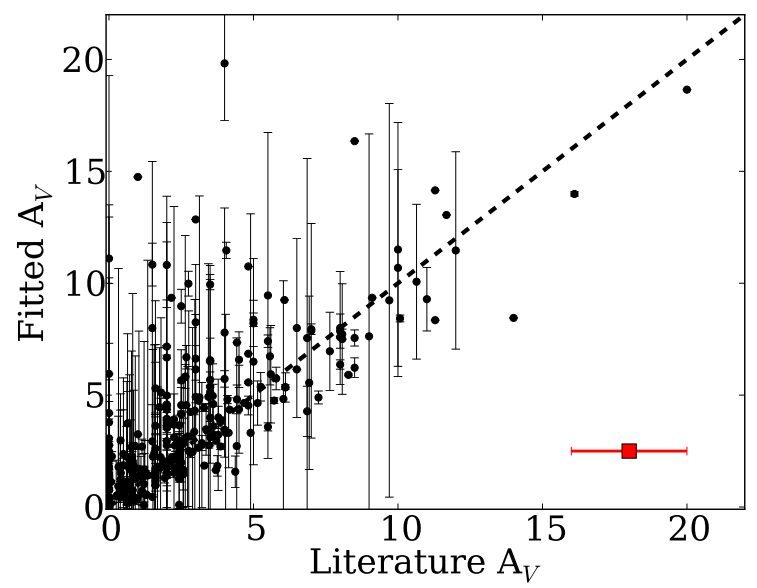

Fig. 1. Comparison between the obtained $A_{\mathrm{V}}$ and the values in the literature. The red square shows the typical uncertainty of spectroscopic $A_{\mathrm{V}}$ estimates from the literature. The identity relation is represented as a dashed line to guide the eye.

only one measurements was available and the $A_{\mathrm{V}}$ could not be properly fitted. After this cleaning process, the final sample is comprised of 2340 objects.

Figure 1 compares the results of the $A_{\mathrm{V}}$ fitting process with the values from the literature when available. In general, our measurements are in agreement with previous estimates within the large uncertainties. Note that these estimates and uncertainties are only tentative. Both our measurements and the measurements from the literature are affected by a number of physical and observational biases (variability, accretion, excesses, photometric errors, rotation, etc.), which cannot be accounted for. The dispersion in Fig. 1 gives a more realistic estimate of the real uncertainties on the $A_{\mathrm{V}}$.

\subsection{Discrepancy between Spitzer and WISE data}

A discrepancy between Spitzer and WISE data was identified during the visual inspection process. We interpret it as the result of contamination from extended background emission and/or source confusion. A more detailed discussion on this issue is given in Appendix A. Data from the AKARI mission (with PSF sizes larger than those of Spitzer and WISE) were also discarded for the same reason.

We therefore discarded all the WISE measurements except for nearby associations from the SACY survey. Given their proximity $(<100 \mathrm{pc})$, the WISE photometry should not be affected by either extended emission (which is rare in these evolved associations) or confusion (also unlikely, since these associations are extremely lose and extended). Visual inspection of the corresponding SEDs and comparison with Spitzer photometry when available confirms that the WISE measurements of SACY sources are indeed not affected by any of these issues, but objects flagged as extended in the WISE catalog were conservatively discarded anyway.

\section{Disk fractions}

In this section we study disk fractions as probed by mid-IR excesses. For the rest of this work, any object with excess at any wavelength longer than $3 \mu \mathrm{m}$ is considered as a disk-bearing system. Prior to identifying disks and deriving disk percentages, we investigated the sensitivity and spatial completeness of the various mid-IR surveys. 
A. Ribas et al.: Disk evolution in the solar neighborhood. I.

Table 2. Sensitivity completeness limits for the Spitzer photometric data.

\begin{tabular}{lccccc}
\hline \hline Regions & IRAC1 (mJy) & IRAC2 (mJy) & IRAC3 (mJy) & IRAC4 (mJy) & MIPS1 (mJy) \\
\hline All regions (except Taurus) & 0.08 & 0.05 & 0.12 & 0.16 & 1.03 \\
Taurus (Rebull et al. 2011) & 0.18 & 0.12 & 0.62 & 0.62 & 5.12 \\
\hline
\end{tabular}

\subsection{Sensitivity completeness}

Circumstellar disks produce a flux excess at IR wavelengths, and hence disk-bearing stars are easier to detect than pure photospheres. A magnitude limited sample such as the one presented here can thus be biased toward objects with disks, leading to overestimated disk fractions. We corrected from this bias by discarding objects with photospheric flux values (as obtained from models used during the SED fitting, see Sect. 4.1) below the detection limits of the different instruments and observations. According to the user manual, the WISE mission (used for the SACY sample) surveyed the whole sky with $5 \sigma$ sensitivity limits of $0.08,0.11,1$ and $6 \mathrm{mJy}$ for the W1, W2, W3, and W4 bands respectively. We estimate that the $\mathrm{W} 1, \mathrm{~W} 2$ and $\mathrm{W} 3$ sensitivities encompass the entire luminosity range of the SACY sample, while the W4 band is sensitive down to the late-K/late-M dwarfs, depending on the age and distance. Spitzer data (used for the rest of the associations, see Table 1) come from various surveys with different sensitivities, summarized in Table 2 . While the optical, near-IR and IRAC data are sensitive to the entire luminosity range described in Sect. 2.2, the shallower MIPS observations define a more limited completeness domain. We estimate that the MIPS sensitivity described in Table 2 corresponds to early-M/early-K dwarfs depending on the age and distance, respectively.

\subsection{Spatial coverage}

WISE is an all-sky survey, and hence the SACY regions - for which only WISE data was used - were fully covered in the mid-IR. On the other hand, Spitzer observations used in this study are covering only fractions of the various associations, resulting in a number of nondetections. These nondetections were naturally rejected in the previous step.

\subsection{Establishing the presence of disks}

Circumstellar disks produce mid/far IR excess in relation to photospheres (Williams \& Cieza 2011). Dust around stars is heated by stellar radiation and re-radiates with typical $T_{\text {eff }}$ of a few tens or hundreds of kelvin. The amount of excess as a function of wavelength depends on the disk properties, resulting in a wide diversity of SED shapes and making the detection of circumstellar disks sometimes difficult using a single color or luminosity.

To identify excesses, we use the significance index $\chi_{\lambda}$, defined as $\chi_{\lambda}=\left(F_{\text {observed }, \lambda}-F_{\text {model }}, \lambda\right) / \sigma_{\text {Observed }, \lambda}$, where $F_{\text {observed }, \lambda}$ is the observed flux, $F_{\text {model }, \lambda}$ is the corresponding model photospheric flux, and $\sigma_{\text {Observed, } \lambda}$ is the error at the corresponding wavelength $(\lambda)$. We define as "excess" any measurement displaying a $\chi_{\lambda} \geq 5$, corresponding to a $5 \sigma$ detection over the expected photospheric value. We note that flux uncertainties play an important role in the definition of $\chi$ and were therefore considered with caution (see Sect. 3.2). Subestimated errors would indeed result in artificially high disk percentages, while overestimated error would result in artificially low disk fractions.
Table 3. Region of the disk probed by the three wavelength ranges.

\begin{tabular}{lcc}
\hline $\begin{array}{l}\text { Wavelength range } \\
(\mu \mathrm{m})\end{array}$ & $\begin{array}{c}\text { Disk radius } \\
(\mathrm{AU})\end{array}$ & Label \\
\hline $3.4-4.6$ & $0.01-1$ & Short \\
$8-12$ & $0.03-5$ & Intermediate \\
$22-24$ & $0.3-60$ & Long \\
\hline
\end{tabular}

To investigate the dependence of the presence of excess as a function of wavelength, we defined three wavelength regimes:

- short wavelength range: sources displaying excess in any band between 3.4-4.6 $\mu \mathrm{m}$ (including IRAC1, IRAC2, WISE1, WISE2);

- intermediate wavelength range: sources displaying excess in any band between $8.0-12.0 \mu \mathrm{m}$ (including IRAC4, WISE3);

- long wavelength range: sources displaying excess in any band between 22-24 $\mu \mathrm{m}$ (including MIPS1 and WISE4).

The mid-IR emission at different wavelengths probes the disk at different distances from the host star and depth within the disk. Table 3 gives an overview of the spatial domains probed by the three wavelength ranges defined above. These values, based on simplifications, should be regarded as indicative only. They nevertheless provide reasonable estimates of the origin of most of the corresponding emission.

Mid-IR excess related to the presence of disks have often been identified using color-color and color-magnitude diagrams. To compare our results with these studies, we also computed fractions for objects detected in all IRAC bands and displaying excess in at least one of them (all regions but SACY) or objects detected in all W1, W2, and W3 bands and excess in any of these three bands (SACY associations).

The "excess" definition based on a conservative $5 \sigma$ level of significance might not be optimized for the detection of debris disks. These evolved disks typically display significantly weaker excesses compared to protoplanetary disks (Wyatt 2008). The corresponding debris disk fractions should therefore be regarded as estimates within the boundaries set by the definition of "excess" used here, rather than absolute values. The same applies for near-IR excesses, these being intrinsically weaker than midIR ones. Our choice of a more conservative threshold ( 5 instead of the "usual" $3 \sigma$ ) is motivated by the problems encountered with the WISE photometry (see Sect. 4.2 and Appendix A), which is used mainly for the older regions and therefore for the objects with the weaker mid-IR excesses. We also note that the usage of two photometric bands in each of the considered wavelength ranges (short, intermediate, long) should mitigate this possible bias.

\subsection{Disk fraction's values and errors}

Disk fractions were calculated as the ratio of objects displaying an excess (as defined before) over the total number of sources after completeness corrections. The large size of our sample 
Table 4. Fraction of sources with excess for each region in the three wavelength regimes.

\begin{tabular}{lcccc}
\hline \hline Name & Fraction ${ }_{\text {IRAC }}(\%)$ & Fraction $_{\text {short }}(\%)$ & Fraction $_{\text {intermediate }}(\%)$ & Fraction $_{\text {long }}(\%)$ \\
\hline 25 Orionis & $9 \pm 5[33]$ & $6 \pm 4[34]$ & $9 \pm 5[33]$ & $\ldots[0]$ \\
Cha I & $52 \pm 6[86]$ & $43 \pm 5[109]$ & $51 \pm 5[102]$ & $66 \pm 9[29]$ \\
Cha II & $84 \pm 9[19]$ & $61 \pm 9[28]$ & $84 \pm 9[19]$ & $87 \pm 9[15]$ \\
CrA & $50 \pm 13[16]$ & $31 \pm 11[19]$ & $50 \pm 13[16]$ & $\ldots[4]$ \\
IC 348 & $36 \pm 3[240]$ & $29 \pm 3[262]$ & $30 \pm 3[240]$ & $50 \pm 17[10]$ \\
$\lambda$-Orionis & $26 \pm 7[43]$ & $20 \pm 6[51]$ & $26 \pm 6[43]$ & $\ldots[0]$ \\
Lupus & $52 \pm 5[85]$ & $38 \pm 5[89]$ & $52 \pm 5[87]$ & $43 \pm 7[49]$ \\
NGC 1333 & $66 \pm 6[67]$ & $47 \pm 6[73]$ & $66 \pm 6[67]$ & $\ldots[2]$ \\
Ophiuchus & $25 \pm 3[214]$ & $17 \pm 3[253]$ & $25 \pm 3[228]$ & $51 \pm 8[41]$ \\
$\sigma$-Orionis & $39 \pm 6[70]$ & $18 \pm 5[74]$ & $39 \pm 6[70]$ & $\ldots[0]$ \\
Serpens & $62 \pm 4[125]$ & $44 \pm 4[131]$ & $60 \pm 5[125]$ & $32 \pm 11[29]$ \\
Taurus & $63 \pm 4[180]$ & $48 \pm 3[212]$ & $63 \pm 4[183]$ & $58 \pm 14[12]$ \\
Upper Sco & $16 \pm 6[32]$ & $7 \pm 2[250]$ & $11 \pm 2[232]$ & $22 \pm 3[165]$ \\
\hline AB Dor & $5 \pm 3[78]$ & $4 \pm 3[78]$ & $3 \pm 2[77]$ & $4 \pm 3[51]$ \\
Argus & $4 \pm 5[44]$ & $0 \pm 3[44]$ & $5 \pm 5[44]$ & $\ldots[3]$ \\
$\beta$ Pic & $18 \pm 6[50]$ & $16 \pm 5[50]$ & $8 \pm 4[59]$ & $22 \pm 6[46]$ \\
Carina & $4 \pm 6[27]$ & $0 \pm 3[27]$ & $4 \pm 5[28]$ & $\ldots[6]$ \\
Columba & $5 \pm 4[57]$ & $2 \pm 2[57]$ & $5 \pm 4[57]$ & $28 \pm 10[18]$ \\
$\eta$ Cha & $21 \pm 9[24]$ & $17 \pm 8[24]$ & $21 \pm 9[24]$ & $30 \pm 15[10]$ \\
Octantis & $6 \pm 7[17]$ & $0 \pm 4[17]$ & $6 \pm 7[17]$ & $\ldots[1]$ \\
Tuc-Hor & $8 \pm 4[48]$ & $8 \pm 4[48]$ & $0 \pm 3[48]$ & $12 \pm 5[41]$ \\
TW Hya & $20 \pm 9[19]$ & $5 \pm 6[19]$ & $16 \pm 8[19]$ & $29 \pm 12[14]$ \\
\hline
\end{tabular}

Notes. The number of sources used is indicated within brackets. Subscripts reference fractions at different wavelength ranges, as defined in Sect. 5.3.

allowed us to perform this analysis for the three wavelength ranges defined previously.

Estimating uncertainties on disk fractions using standard error propagation is impractical, as uncertainties on the photometry (errors related to measurement, but also variability, rotation, activity, etc.), the fit $\left(A_{\mathrm{V}}\right)$ and various models (adopted extinction law, Spectral Type vs. $T_{\text {eff }}$ relation) are difficult to assess. We chose to estimate errors in an empirical way by using bootstrapping (1000 iterations for each region) and randomly varying the $T_{\text {eff }}$ and observed fluxes within normal distributions (with $\sigma$ of $100 \mathrm{~K}$ and $\sigma_{\text {flux }}$, respectively). For regions with a high number of sources $(>30)$, disk fraction errors are set as the standard deviation obtained with the boostrapping process. For smaller samples, the errors were corrected using the corresponding Student's $t$ distribution with a confidence interval of $68 \%$ (comparable to the $1 \sigma$ value for larger samples). The derived disk fraction values are given in Table 4 and Figs. 2 and 3. Because of the different sensitivity and spatial completeness levels of the sample, the number of sources varies in each bands. To avoid problems related to small number statistics, we discarded datasets with less than ten objects per region and wavelength range.

\section{Disk fractions as a function of age}

In this section, we investigate disk dissipation timescales as a function of age and wavelength. As a sanity check, we first compare our results with previous studies from Hernández et al. (2007b) and Hernández et al. (2008) for the nine star-forming regions in common: 25 Orionis, Cha I, $\eta$-Cha, IC 348, $\lambda$-Orionis, NGC 1333, $\sigma$-Orionis, Taurus and Upper Sco. Figure 2 shows that the results are in good agreement within the estimated uncertainties. We also compare our results for part of the SACY sample from those in Zuckerman et al. (2011): all but one (AB Dor) associations are within a $2 \sigma$ range. We note that our values are almost systematically lower than the ones given in the literature, as expected given the more conservative disk detection criterion used in this work (see Sect. 5.3).

We then study the excess fraction for each of the three wavelength ranges as a function of age (Fig. 2, top right and lower panels). Our results show a decay of disk fractions with age at all wavelength ranges The values obtained for Cha II are considerably higher than those of other associations of similar age, but consistent with the value reported by Alcalá et al. (2008). The current dataset does not allow us to explain this difference, and the cause is outside the scope of this study. Figure 2 shows that disk fractions for the intermediate $(8-12 \mu \mathrm{m})$ wavelength range are higher than for the short $(3.4-4.6 \mu \mathrm{m})$ wavelength range. Although of low statistical significance (within $1 \sigma$ ), the systematic offset for the entire sample suggests that this relative difference is a real trend.

In this study, we follow Mamajek (2009) and model these distributions with an exponential law of the form:

$A \mathrm{e}^{-t / \tau}+C$

where $t$ is the age of the region (in Myr), and $A, \tau$ and $C$ are left as free parameters. In this simple parametric model, $\tau$ can be interpreted as the characteristic timescale of IR excess decay, and has been previously found to have values between $2 \sim 3 \mathrm{Myr}$ (Mamajek 2009; Williams \& Cieza 2011). The best fits obtained for $\tau$ in the short $(3.4-4.6 \mu \mathrm{m})$ and intermediate $(8-12 \mu \mathrm{m})$ wavelength ranges are $2.3 \pm 0.5 \mathrm{Myr}$ and $2.8 \pm 0.8 \mathrm{Myr}$ respectively, indistinguishable within the uncertainties and in good agreement with the results from Mamajek (2009). The exponential fit has the advantage of modeling the entire time domain with a single function, and was therefore preferred over the linear fit model used in Haisch et al. (2001). Further investigation of the possible analytic laws to model this behavior and their corresponding physical interpretation are outside the scope of our work.

Whereas IR excesses in the short and intermediate wavelength ranges are thought to come from primordial opticallythick disks (Williams \& Cieza 2011), excesses in the long 
A. Ribas et al.: Disk evolution in the solar neighborhood. I.
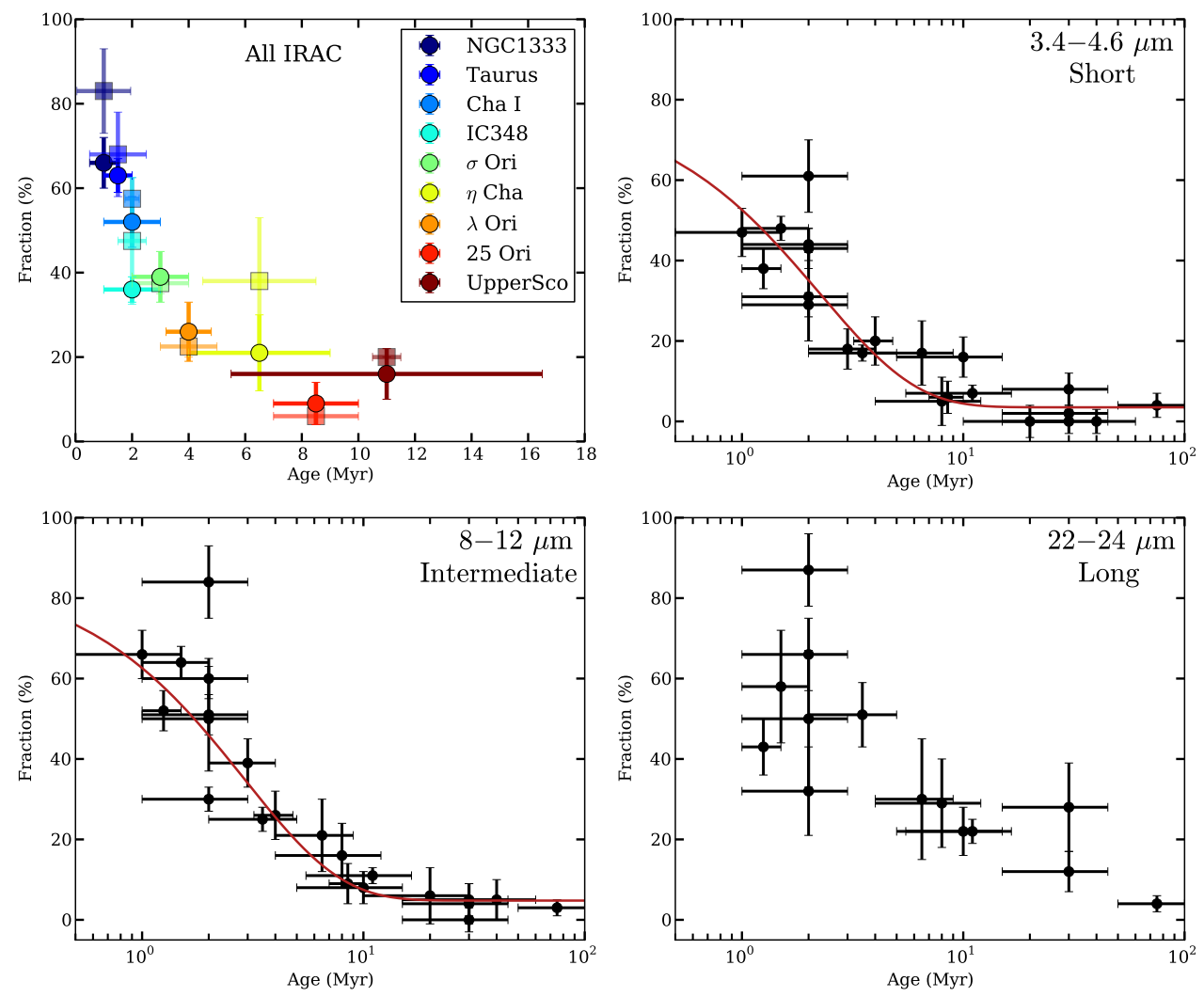

Fig. 2. Upper left panel: comparison of the disk fractions obtained in this study (circles) with those from Hernández et al. (2007b, 2008) (squares) for regions in common. Upper right and lower panels: disk fractions for all regions in the short, intermediate and long wavelength ranges. The best fit exponential law is over-plotted (red line).

wavelength range $(22-24 \mu \mathrm{m})$ can be produced by either primordial, transitional or debris disks (Wyatt 2008). These different types of disk do not necessarily have the same evolutionary mechanisms and timescales (Wyatt 2008). Fitting a simple exponential law to the long wavelength dataset would therefore have no physical meaning and was not attempted here.

To disentangle disks populations in the long wavelength dataset, we split the sample in two groups:

_ "primordial" disks: sources with excess at 22-24 $\mu \mathrm{m}$ and at least one shorter wavelength;

- "evolved" disks: sources with excess at 22-24 $\mu \mathrm{m}$ only, including mostly transitional and debris disks. In some cases, edge-on disks can mimic the SED of transitional disks and contaminate the group. Their frequency is nevertheless expected to be very low.

Figure 3 shows the fractions of primordial and evolved disks as a function of time. The distribution is more dispersed than those found at shorter wavelengths (Fig. 2), specially at very young ages $(<2 \mathrm{Myr})$. After $2 \sim 3 \mathrm{Myr}$, it shows a clear and smooth decay and reaches a $\sim$ zero level around $10 \sim 30 \mathrm{Myr}$, as observed at shorter wavelengths. To estimate the timescale of excess decay, we fit an exponential law to:

1. the whole primordial disks dataset: $\tau=5.5 \pm 1.4 \mathrm{Myr}$;

2. the highest values at every given age: $\tau=4.2 \pm 0.6 \mathrm{Myr}$;

3 . the lowest value at every given age: $\tau=5.8 \pm 1.5 \mathrm{Myr}$.

In all three cases - including the extremes $-\tau$ is significantly higher than in the short and intermediate wavelength ranges. Fitted values with their estimated errors for the short,

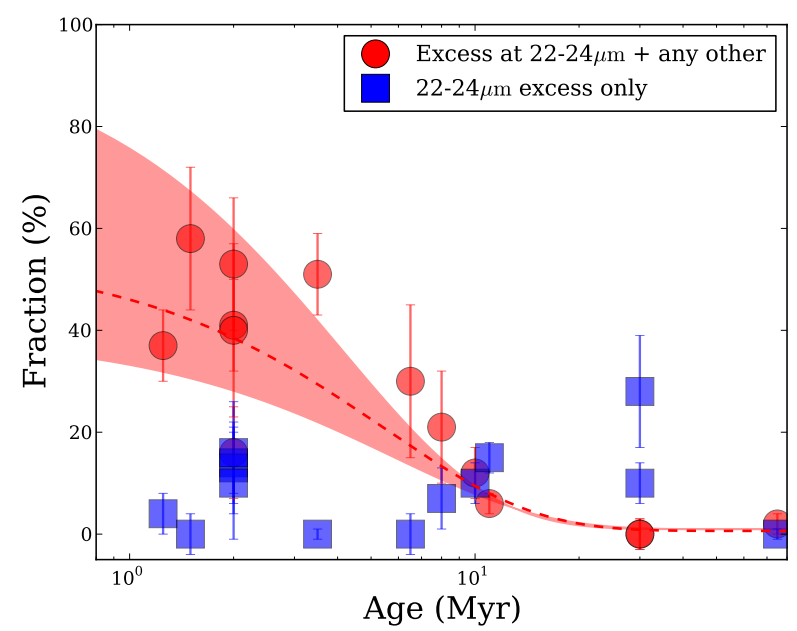

Fig. 3. Fraction of primordial (red circles) and evolved (blue squares) disks as a function of age. The best fit exponential law for the primordial disk percentages is overplotted as a red line. The red shaded area represents the fit obtained for two extreme cases: the highest and lowest values at every age.

intermediate, and "primordial" long wavelength regimes are given in Table 5.

\section{Discussion}

\subsection{Wavelength-dependent disk fractions}

The unprecedented size of our sample and the consistency of our analysis allows for the first time to perform statistically 
Table 5. Values and errors obtained from fitting and exponential decay to disk fractions with time.

\begin{tabular}{lccc}
\hline \hline Wavelength range & $\begin{array}{c}\mathrm{A} \\
(\% / \mathrm{Myr})\end{array}$ & $\begin{array}{c}\tau \\
(\mathrm{Myr})\end{array}$ & $\begin{array}{c}\mathrm{C} \\
(\%)\end{array}$ \\
\hline Short & $80 \pm 10$ & $2.3 \pm 0.5$ & $4 \pm 2$ \\
Intermediate & $80 \pm 10$ & $2.8 \pm 0.8$ & $5 \pm 2$ \\
Long ("primordial") & $40-100$ & $4.2-5.8$ & $0-2$ \\
\hline
\end{tabular}

Notes. In the case of long wavelength range, only "primordial" disks were fitted, and hence no value is provided for "evolved" disks (see Sect. 6).

robust measurements of disk fractions as a function of both time and wavelength. Figure 2 shows that below $10 \mathrm{Myr}$, disk fractions derived from mid-IR excess systematically increase with wavelength (as shown in Table 4). We note that this difference could be affected by the stringent "excess" criterion applied here, which could favor the detection of strong excesses typical of mid-IR bands. We verify that the trend of increasing disk fractions with wavelength up to $10 \mathrm{Myr}$ is still present when a $\chi>3$ cut is used. This result suggests that great care should be taken when comparing studies of disk fractions derived using different wavelength ranges.

\subsection{Disk decay and dust evolution}

The timescale of mid-IR excess decay obtained in the short and intermediate wavelength ranges are found to be similar, suggesting that the corresponding dust populations evolve on a similar timescale. One the other hand, Fig. 2 shows that there is more excess at intermediate wavelengths at a given age than at short wavelengths. The two results combined suggest that the dust emitting at shorter wavelengths - which is in average closer to the star - starts evolving sooner.

The higher mid-IR excess fractions at all ages and the longer decay times scale in the long wavelength range $(22-24 \mu \mathrm{m})$ both suggest that the dust at larger radii evolves slower. Although marginal, the higher dispersion of the excesses distribution at long wavelengths points toward a less coherent evolution of the dust in that regime than at shorter wavelengths. The dust populations at larger radii are indeed believed to experience both vertical (settling) and radial (mixing, migration, etc.) transfers during the disk evolution. Grain growth, leading eventually to planetary embryos, is also affecting the disk properties and could participate to the observed scatter in time.

\subsection{Transition from protoplanetary to debris disks}

Figure 4 shows flux ratios (observed over expected photospheric values) in the long wavelength range $(22-24 \mu \mathrm{m})$ for all objects as a function of age. Primordial disks display stronger excesses over the photosphere than their evolved counterparts. A sharp decline in flux excess is observed around $10 \mathrm{Myr}$, indicating a significant change in the disk properties at that stage. On the other hand, Figs. 2 and 3 show that disk fractions reach $\mathrm{a} \sim$ zero level at about the same time. These two results confirm the disk lifetime of $\approx 10 \mathrm{Myr}$ reported previously in the literature (Haisch et al. 2001; Hernández et al. 2007b, 2008; Mamajek 2009; Williams \& Cieza 2011). Unfortunately, current data do not provide information on whether the material found in young debris disks are remnants of the protoplanetary stage, or it is a different dust population produced by planetesimal collisions

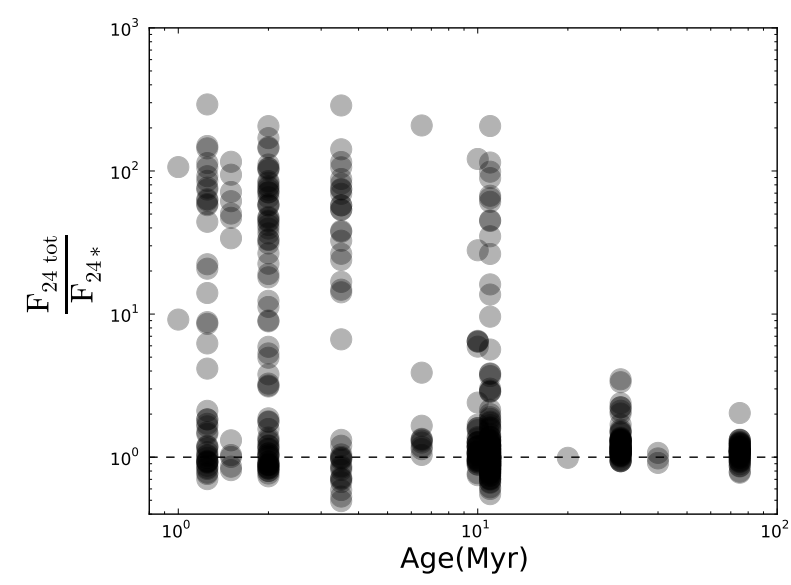

Fig. 4. Ratios of the observed and predicted photospheric values in the long wavelength range $(22-24 \mu \mathrm{m})$ as a function of age.

(Wyatt 2008). Dissecting this transition from the gas rich to gas poor phases is one of the overall long-term goals of this project.

Tendencies for "evolved" disks are much harder to determine given their low number at all ages. However, two interesting features can be identified in Fig. 3. First, "evolved" disks are not present (or extremely uncommon) at young ages ( $\$ 5 \mathrm{Myr}$ ). Second, the fraction of "evolved" disk seems to show a bump starting at $\sim 8 \mathrm{Myr}$, matching the disappearance of the "primordial" disks. The latter feature, which needs to be confirmed with a larger sample, reproduces the results by Currie et al. (2008) who reported an increase of mid-IR emission from debris disks at 5-10 Myr, followed by a peak at 10-15 Myr, and a decrease from there on in the double cluster $\mathrm{h}$ and $\chi$ Persei. They argue that this qualitatively matches the predictions from the debris disk evolution tracks by Kenyon \& Bromley (2004) for disk masses between 3 and 1/3 of the Minimum Mass Solar Nebula, and that kilometer-sized planetesimals already formed between 0 and 2 Myr. This suggest a disk self-stirring process (Wyatt 2008), which would fill the system with dust produced by collisions of planetesimals and increase the mid-IR flux for some Myr at 22-24 $\mu \mathrm{m}$. As a result, a higher debris disk detection rate will be obtained, and hence higher disk numbers. The combination of these two features (no "evolved" disks at young ages, plus their fractions tentatively increasing when "primordial" disks start disappearing) reinforces the idea of "evolved" disks being the outcome of "primordial" disk evolution. Interestingly, the disappearance of "primordial" disks, and hence gas (Sicilia-Aguilar et al. 2005; Sicilia-Aguilar et al. 2006), seems to match in time the period of dust stirring, which indeed require negligible gas to be effective (Wyatt 2008).

\section{Conclusions}

We have compiled a large sample of 2340 spectroscopically confirmed young stellar objects from 22 nearby $(<500$ pc) associations. Spectral energy distributions covering from visible to mid-IR (22-24 $\mu \mathrm{m})$ wavelengths have been built and visually inspected for all the sources. We studied the disk fraction as probed by excess over the photosphere in three wavelength ranges: 3.4-4.6 $\mu \mathrm{m}$ (short), 8-12 $\mu \mathrm{m}$ (intermediate) and 22-22 $\mu \mathrm{m}$ (long), and find:

- The excess fraction is dependent on the wavelength range considered. Transforming excess percentages to disk percentages is therefore not straightforward, and special care 
must be taken when comparing disk fractions derived using differing wavelengths.

- Significant discrepancies between the Spitzer and WISE photometry are found, mostly due to confusion and extended background emission, and calling for a revision of previous studies based on blind analysis of the WISE luminosities.

- Mid-IR excesses are systematically more frequent at longer wavelength, compatible with inside-out disk clearing scenarios.

- For primordial disks, the dust probed between 22-24 $\mu \mathrm{m}$ seems to evolve more slowly. Assuming an exponential decay, we derive a timescale $\tau$ of $4 \sim 6 \mathrm{Myr}$ at 22-24 $\mu \mathrm{m}$, compared to $2 \sim 3 \mathrm{Myr}$ at shorter wavelengths. This result is also compatible with inside-out disk clearing.

- There is more dispersion in the fraction of excess sources with age when measured at $22-24 \mu \mathrm{m}$ in comparison to shorter wavelengths, suggesting that other mechanisms are involved in the evolution of the dust at larger radii.

- The fraction of "evolved" disks seems to rise around 8-10 Myr, and decrease again afterward.

- The disappearance of "primordial" (potentially gas-rich) disks at $10 \sim 20 \mathrm{Myr}$ matches in time the brief rise of the number of "evolved" disks. This result is compatible with planet formation theories suggesting that the disappearance of the gas is immediately followed by the dynamical stirring of the debris disk produced by planetesimal collisions that will ultimately lead to planet formation.

- These last two conclusions reinforce the idea of "evolved" disks being the outcome of "primordial" disk evolution.

Future studies based on the present sample will extend these results, obtained for the integrated stellar mass spectrum, by investigating disk fractions and properties as a function of stellar mass, age, and environment. The inclusion of far-IR data from Herschel will ultimately provide crucial information about outer disk regions and disk masses, contributing to a much more complete picture of disk evolution.

\section{References}

Alcalá, J. M., Spezzi, L., Chapman, N., et al. 2008, ApJ, 676, 427

Allard, F., Homeier, D., \& Freytag, B. 2012, Roy. Soc. London Philos. Trans. Ser. A, 370, 2765

Allen, P. R., Luhman, K. L., Myers, P. C., et al. 2007, ApJ, 655, 1095

Alves de Oliveira, C., Moraux, E., Bouvier, J., et al. 2010, A\&A, 515, A75

Alves de Oliveira, C., Moraux, E., Bouvier, J., et al. 2013, A\&A, 549, A123

Barrado y Navascués, D., Béjar, V. J. S., Mundt, R., et al. 2003, A\&A, 404, 171

Barrado y Navascués, D., Stauffer, J. R., Morales-Calderón, M., et al. 2007, ApJ, 664,481

Bayo, A., Barrado, D., Stauffer, J., et al. 2011, A\&A, 536, A63

Bell, C. P. M., Naylor, T., Mayne, N. J., Jeffries, R. D., \& Littlefair, S. P. 2013, MNRAS, 34, 806

Caballero, J. A. 2007, A\&A, 466, 917

Carpenter, J. M., Mamajek, E. E., Hillenbrand, L. A., \& Meyer, M. R. 2006, ApJ, 651, L49

Comerón, F. 2008, in Handbook of star forming regions, vol. II, ed. B. Reipurth (ASP Monograph Publ.), 295

Currie, T., Kenyon, S. J., Balog, Z., et al. 2008, ApJ, 672, 558

DENIS Consortium 2005, VizieR Online Data Catalog: II/263

Duchêne, G. \& Kraus, A. 2013, ARA\&A, 51, 269

Erickson, K. L., Wilking, B. A., Meyer, M. R., Robinson, J. G., \& Stephenson, L. N. 2011, AJ, 142, 140

Evans, II, N. J., Dunham, M. M., Jørgensen, J. K., et al. 2009, ApJS, 181, 321 Franciosini, E., Pallavicini, R., \& Sanz-Forcada, J. 2006, A\&A, 446, 501 Gullbring, E., Calvet, N., Muzerolle, J., \& Hartmann, L. 2000, ApJ, 544, 927
Gutermuth, R. A., Myers, P. C., Megeath, S. T., et al. 2008, ApJ, 674, 336 Haisch, Jr., K. E., Lada, E. A., \& Lada, C. J. 2001, ApJ, 553, L153 Hartmann, L. 2001, AJ, 121, 1030

Hartmann, L., Hewett, R., \& Calvet, N. 1994, ApJ, 426, 669

Hernández, J., Calvet, N., Briceño, C., et al. 2007a, ApJ, 671, 1784

Hernández, J., Hartmann, L., Megeath, T., et al. 2007b, ApJ, 662, 1067

Hernández, J., Hartmann, L., Calvet, N., et al. 2008, ApJ, 686, 1195

Hirota, T., Bushimata, T., Choi, Y. K., et al. 2008, PASJ, 60, 37

Høg, E., Fabricius, C., Makarov, V. V., et al. 2000, A\&A, 355, L27

Indebetouw, R., Mathis, J. S., Babler, B. L., et al. 2005, ApJ, 619, 931

Ishihara, D., Onaka, T., Kataza, H., et al. 2010, A\&A, 514, A1

Kenyon, S. J., \& Bromley, B. C. 2004, AJ, 127, 513

Krautter, J., Wichmann, R., Schmitt, J. H. M. M., et al. 1997, A\&AS, 123, 329

Lada, C. J. 2006, ApJ, 640, L63

Lada, C. J., Muench, A. A., Luhman, K. L., et al. 2006, AJ, 131, 1574

Lodieu, N., Dobbie, P. D., \& Hambly, N. C. 2011, A\&A, 527, A24

Luhman, K. L. 2004, ApJ, 617, 1216

Luhman, K. L. 2007, ApJS, 173, 104

Luhman, K. L., \& Muench, A. A. 2008, ApJ, 684, 654

Luhman, K. L., Stauffer, J. R., Muench, A. A., et al. 2003, ApJ, 593, 1093

Luhman, K. L., Allen, L. E., Allen, P. R., et al. 2008a, ApJ, 675, 1375

Luhman, K. L., Hernández, J., Downes, J. J., Hartmann, L., \& Briceño, C. 2008b, ApJ, 688, 362

Mamajek, E. E. 2009, in AIP Conf. Ser. 1158, eds. T. Usuda, M. Tamura, \& M. Ishii, 3

Monin, J.-L., Guieu, S., Pinte, C., et al. 2010, A\&A, 515, A91

Mortier, A., Oliveira, I., \& van Dishoeck, E. F. 2011, MNRAS, 418, 1194

Murakami, H., Baba, H., Barthel, P., et al. 2007, PASJ, 59, 369

Muzerolle, J., Hillenbrand, L., Calvet, N., Briceño, C., \& Hartmann, L. 2003, ApJ, 592, 266

Natta, A., Testi, L., Comerón, F., et al. 2002, A\&A, 393, 597

Neuhäuser, R., Walter, F. M., Covino, E., et al. 2000, A\&AS, 146, 323

Nisini, B., Antoniucci, S., Giannini, T., \& Lorenzetti, D. 2005, A\&A, 429, 543

Oliveira, I., Merín, B., Pontoppidan, K. M., et al. 2009, ApJ, 691, 672

Pecaut, M. J., Mamajek, E. E., \& Bubar, E. J. 2012, ApJ, 746, 154

Perryman, M. A. C., Lindegren, L., Kovalevsky, J., et al. 1997, A\&A, 323, L49

Peterson, D. E., Caratti o Garatti, A., Bourke, T. L., et al. 2011, ApJS, 194, 43

Preibisch, T., Brown, A. G. A., Bridges, T., Guenther, E., \& Zinnecker, H. 2002, AJ, 124, 404

Rebull, L. M., Padgett, D. L., McCabe, C.-E., et al. 2010, ApJS, 186, 259

Rebull, L. M., Koenig, X. P., Padgett, D. L., et al. 2011, ApJS, 196, 4

Reipurth, B. 2008a, Handbook of Star Forming Regions, Vol. I: The Northern Sky (ASP Monograph Publ.), 4

Reipurth, B. 2008b, Handbook of Star Forming Regions, Vol. II: The Southern Sky (ASP Monograph Publ.), 5

Rigliaco, E., Natta, A., Testi, L., et al. 2012, A\&A, 548, A56

Sacco, G. G., Franciosini, E., Randich, S., \& Pallavicini, R. 2008, A\&A, 488, 167

Schmidt-Kaler, T. 1982, in Landolt-Bornstein, Group VI, ed. K.-H. Hellwege (Berlin: Springer), 454, 2

Shu, F., Najita, J., Ostriker, E., et al. 1994, ApJ, 429, 781

Sicilia-Aguilar, A., Hartmann, L. W., Hernández, J., Briceño, C., \& Calvet, N. 2005, AJ, 130, 188

Sicilia-Aguilar, A., Hartmann, L., Calvet, N., et al. 2006, ApJ, 638, 897

Sicilia-Aguilar, A., Henning, T., Juhász, A., et al. 2008, ApJ, 687, 1145

Sicilia-Aguilar, A., Henning, T., Kainulainen, J., \& Roccatagliata, V. 2011, ApJ 736, 137

Skrutskie, M. F., Cutri, R. M., Stiening, R., et al. 2006, AJ, 131, 1163

Soderblom, D. R., Hillenbrand, L. A., Jeffries, R. D., Mamajek, E. E., \& Naylor T. 2013, Protostars and Planets VI (University of Arizona Press), accepted

Spezzi, L., Alcalá, J. M., Covino, E., et al. 2008, ApJ, 680, 1295

Taylor, M. B. 2005, in Astronomical Data Analysis Software and Systems XIV, eds. P. Shopbell, M. Britton, \& R. Ebert, ASP Conf. Ser., 347, 29

Torres, C. A. O., Quast, G. R., Melo, C. H. F., \& Sterzik, M. F. 2008, Young Nearby Loose Associations, ed. B. Reipurth, 5, 757

Wilking, B. A., Meyer, M. R., Robinson, J. G., \& Greene, T. P. 2005, AJ, 130, 1733

Williams, J. P., \& Cieza, L. A. 2011, ARA\&A, 49, 67

Winston, E., Megeath, S. T., Wolk, S. J., et al. 2009, AJ, 137, 4777

Wright, E. L., Eisenhardt, P. R. M., Mainzer, A. K., et al. 2010, AJ, 140, 1868

Wyatt, M. C. 2008, ARA\&A, 46, 339

Zapatero Osorio, M. R., Béjar, V. J. S., Pavlenko, Y., et al. 2002, A\&A, 384, 937

Zuckerman, B., Rhee, J. H., Song, I., \& Bessell, M. S. 2011, ApJ, 732, 61

Pages 10 to 11 are available in the electronic edition of the journal at http://www . aanda.org 
Acknowledgements. This work has been possible thanks to the ESAC Science Operations Division research funds with code SC 1300016149, support from the ESAC Space Science Faculty and of the Herschel Science Centre. We thank the referee for valuable comments that helped to improve the paper, as well as Eric Mamajek for additional suggestions. We also want to thank Neal J. Evans II, Ewine F. van Dishoeck, Paul Harvey, Geoffrey A. Blake, Fernando Comerón, Lucas Cieza, Isa Oliveira, Luisa Rebull, Juan Alcalá, Joanna Brown, Jean-Charles Augereau, Vincent Geers and other members of the $\mathrm{c} 2 \mathrm{~d}$ team for comments on a previous $\mathrm{c} 2 \mathrm{~d}$ works on the same topic that had to be abandoned due to inappropriate data for the intended study. H. Bouy is funded by the Spanish Ramón y Cajal fellowship program number RYC-2009-04497. This work has made an extensive use of Topcat (http://www.star.bristol.ac.uk/ mbt/topcat/, Taylor 2005). This work is based in part on data obtained as part of the UKIRT Infrared Deep Sky Survey. This research made use of the SDSS-III catalog. Funding for SDSS-III has been provided by the Alfred P. Sloan Foundation, the Participating Institutions, the National Science Foundation, and the US Department of Energy Office of Science. The SDSS-III web site is http://www . sdss3.org/. SDSS-III is managed by the Astrophysical Research Consortium for the Participating Institutions of the SDSS-III Collaboration including the University of Arizona, the Brazilian Participation Group, Brookhaven National Laboratory, University of Cambridge, University of Florida, the French Participation Group, the German Participation Group, the Instituto de Astrofísica de Canarias, the Michigan State/Notre Dame/JINA Participation Group, Johns Hopkins University, Lawrence Berkeley National Laboratory, Max Planck Institute for Astrophysics, New Mexico State University, New York University, Ohio State University, Pennsylvania State University, University of Portsmouth, Princeton University, the Spanish Participation Group, University of Tokyo, University of Utah, Vanderbilt University, University of Virginia, University of Washington, and Yale University. This work makes use of data from the DENIS Survey. DENIS is the result of a joint effort involving human and financial contributions of several Institutes mostly located in Europe. It has been supported financially mainly by the French Institut National des Sciences de l'Univers, from CNRS, and the French Education Ministry, the European Southern Observatory, the State of Baden-Württemberg, and the European Commission under networks of the SCIENCE and Human Capital and Mobility programs, the Landessternwarte Heidelberg and the Institut d'Astrophysique de Paris. This publication makes use of data products from the Wide-field Infrared Survey Explorer, which is a joint project of the University of California, Los Angeles, and the Jet Propulsion Laboratory/California Institute of Technology, funded by the National Aeronautics and Space Administration. This research used the facilities of the Canadian Astronomy Data Centre operated by the National Research Council of Canada with the support of the Canadian Space Agency. This publication makes use of data products from the Two Micron All Sky Survey, which is a joint project of the University of Massachusetts and the Infrared Processing and Analysis Center/California Institute of Technology, funded by the National Aeronautics and Space Administration and the National Science
Foundation. This work is based in part on observations made with the Spitzer Space Telescope, which is operated by the Jet Propulsion Laboratory, California Institute of Technology under a contract with NASA.

\section{Appendix A: On the discrepancy between Spitzer and WISE data}

The WISE mission used four IR bands $(3.4,4.6,12$, and $22 \mu \mathrm{m}$, $\mathrm{W} 1, \mathrm{~W} 2, \mathrm{~W} 3$ and $\mathrm{W} 4$ respectively) to survey the whole sky. On the other hand, the Spitzer Space Telescope observed several star-forming regions with its instruments $\operatorname{IRAC}(3.6,4.5$, 5.8, and $8 \mu \mathrm{m}$ ) and MIPS (24, 70 and $160 \mu \mathrm{m}$, the last two not used in this study). IRAC1, IRAC2 and MIPS1 bands are therefore comparable to $\mathrm{W} 1, \mathrm{~W} 2$ and $\mathrm{W} 4$, respectively. We computed the ratio of measured fluxes (as $\mathrm{WISE}_{\text {band }} / \mathrm{IRAC}_{\mathrm{band}}$ ) as a function of $\mathrm{IRAC}_{\mathrm{band}}$ flux, where band corresponds to each of the comparisons mentioned earlier. The results are shown in Fig. A.1: W1 and W2 are compatible with the corresponding IRAC photometry, whereas W4 deviates significantly from MIPS1 measurements. We found this effect to be more important for weaker fluxes, which could indicate two different issues: (1) a problem with WISE flux estimates for unresolved sources, specially important for confused regions; or (2) an important influence of extended background emission. Figures A.1 and A.2 illustrate these two problems in the case of the IC 348 young stellar cluster. IC 348 is a rather dense young cluster with a high and variable background level. WISE fluxes in the $W 3$ and W4 bands were found to be incompatible with the Spitzer ones. The full width at half-maximum (FWHM) of the W4 PSF is 11" (WISE All Sky Release Explanatory Supplement), considerably larger than the 6" FWHM of the MIPS1 PSF (MIPS Instrument Handbooks), resulting in a higher level of confusion and a greater sensitivity to variable extended background. Figure A.3 shows an example of a problematic source within IC 348. If we trust the WISE photometry blindly, a mid-IR excess is found at 12 and $22 \mu \mathrm{m}$. The corresponding Spitzer photometry demonstrates that no such excess exists, and that the source most likely does not harbor a disk. 
A. Ribas et al.: Disk evolution in the solar neighborhood. I.
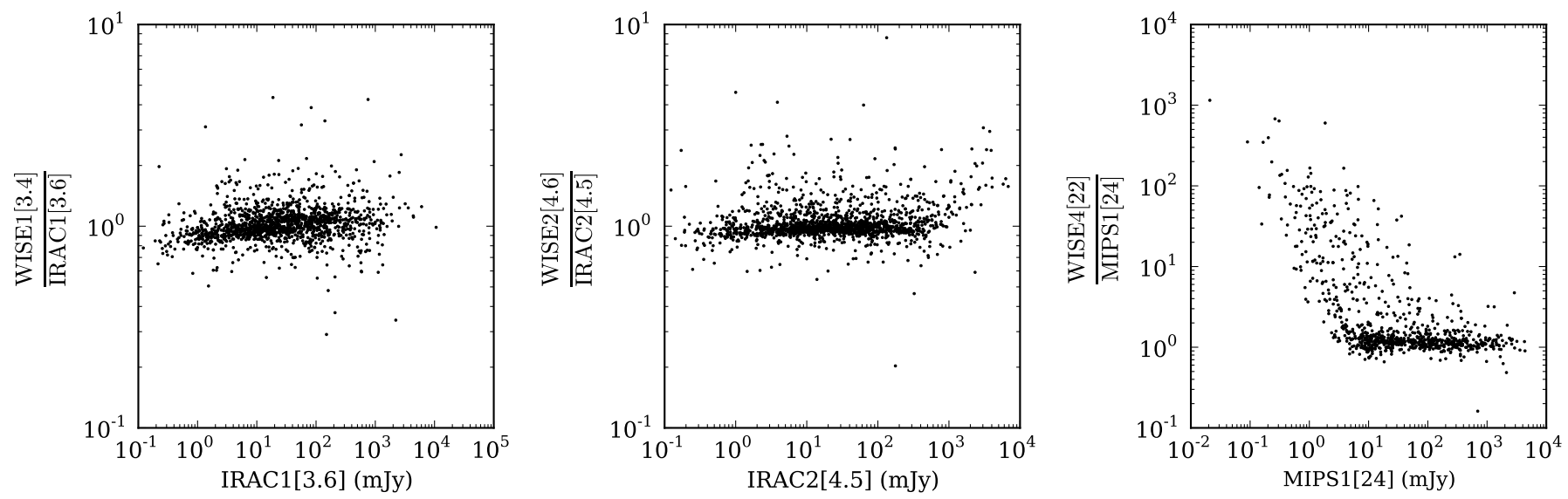

Fig. A.1. Differences between WISE and Spitzer data as a function of Spitzer fluxes for the three comparable bands. Only WISE detections with $S / N>5$ are plotted in each band.
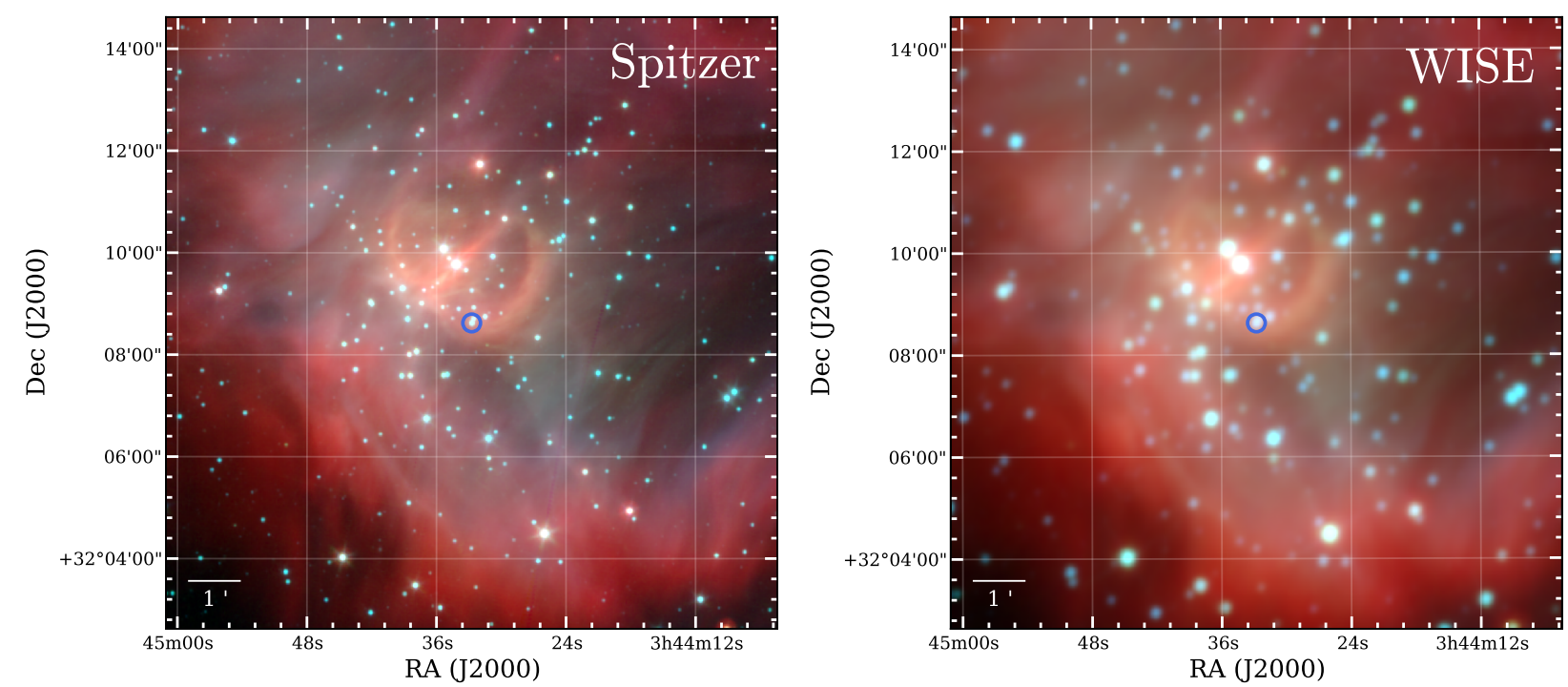

Fig. A.2. Three-color composite images of IC 348 as seen by Spitzer (left, blue: IRAC1, green: IRAC2, and red: MIPS1) and WISE (right, blue: W1, green: W2, and red: W4). The blue dots marks the position of 2MASS J03443274+3208374, which SED is shown in Fig. A.3.

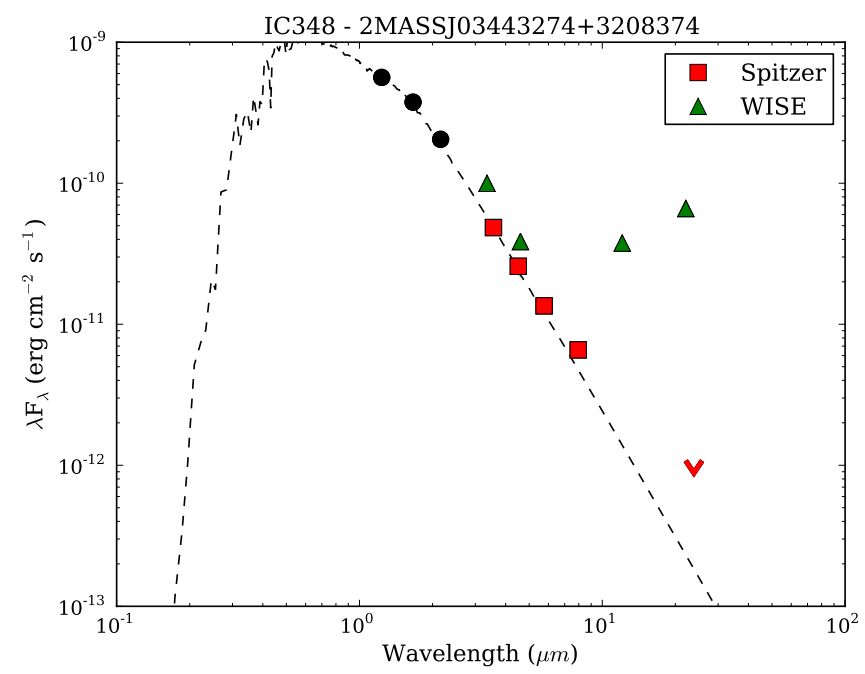

Fig. A.3. SED of 2MASS J03443274+3208374, a source located in a region of high density and variable extended emission (see Fig. A.2). 